Author's reply: Amminger et al raise some interesting issues. I certainly agree that the estimation of premorbid IQ, particularly in patients with schizophrenia, is challenging and that further validation studies on methods for making such estimates should be pursued.

More specifically with reference to our earlier paper on the relationship of DUP to cognitive functioning (Norman et al, 2001), Amminger et al argue for the likely superiority of Bilder et al's (1992) index as a measure of cognitive deterioration in contrast to estimates based on NARTestimated premorbid IQ minus current WAIS full-scale IQ. In this respect they note that $38.1 \%$ of patients in a recent study by their group showed higher current IQ than NART-estimated premorbid IQ. This would, of course, suggest an increase in IQ after illness onset - an unlikely occurrence. I have examined this issue in our data-set and found such a pattern in $17.8 \%$ of our sample, with the average discrepancy among these individuals being 8.4 points. I can also confirm that in our sample, as in Amminger et al's sample, NART scores were correlated with age at admission $(r=0.24, P<0.05)$, but WAIS$\mathrm{R}$ full-scale scores were not.

The substantive question, of course, is whether DUP is related to cognitive deterioration. Amminger et al report that they have found DUP related to deterioration based on Bilder's index. We had reported some results using Bilder's index in our earlier paper. I will take this opportunity to report further that when we examined correlations between our two indices of DUP and Bilder's deterioration index they were non-significant $(r=0.06$ and $r=0.04)$. We are currently pursuing the issue of whether DUP may be related to recovery of cognitive functioning during the first year of treatment.

The discrepancy between our earlier findings and those of Dr Amminger and colleagues does not appear to be explained on the basis of use of the NART rather than Bilder index. Other variables related to sample composition may be relevant. Also of potential importance is the method of measuring DUP, which, as has been suggested elsewhere (Norman \& Malla, 2001), also needs to be more carefully considered and standardised. In this, as in many areas of psychiatric research, cumulative progress is dependent on careful and comparable measurement across studies. I endorse Amminger et al's comments in this respect.

Bilder, R. M., Lipschutz-Broch, L., Reiter, G., et al (1992) Intellectual deficits in first-episode schizophrenia: evidence for progressive deterioration. Schizophrenia Bulletin, 18, 437-448.

Norman, R. M. G. \& Malla, A. K. (200I) Duration of untreated psychosis: a critical examination of the concept and its importance. Psychological Medicine, 31, $381-400$.

_, Townsend, L. \& Malla, A. K. (200I) Duration of untreated psychosis and cognitive functioning in first-episode patients. British Journal of Psychiatry, 179, 340-345

R. M. G. Norman London Health Sciences Centre, Victoria Campus, 375 South Street, London, Ontario, Canada N6A 4G5

\section{Depression: detection and diagnosis}

The October 2001 issue of the Journal reports two prevalence studies of depressive disorders (Ayuso-Mateos et al, 2001; Thompson et al, 2001). Both studies used a self-report questionnaire as an initial screening device although both avoided the sometimes reported but unjustified assertion of diagnosis based upon scores of the scales. Such scales are widely used in the manner reported by these studies and a cautionary comment is in order. There is a widespread view that the selection of instrument is unimportant so long as it is designated as a 'depression' scale; this is not true. For instance, the scales used in the above-mentioned studies were the Beck Depression Inventory (BDI) (in Ayuso-Mateos et al, 2001) and the depression sub-scale of the Hospital Anxiety and Depression scale (HAD-D) (in Thompson et al, 2001). These two instruments highlight very different aspects of depressive disorders (Snaith, 1993). The HAD-D has $86 \%$ of its variance directed to mood symptoms (depressed mood and anhedonia) but an absence of cognitive symptoms (hopelessness, low self-esteem and guilt ideation). With the BDI the reverse is the case, with $14 \%$ directed to mood and/or anhedonia but $33 \%$ focusing on the cognitive symptoms.

There is an unfortunate tendency to refute the importance of difference based upon predominant psychopathology and even, within the realm of depressive disorders, to deny the importance of diagnosis. Indeed, the first study uses the term 'prejudice' when referring to the separation of disorders and frankly advocates the conflation of disorders of major depression (for which one or other of the mood symptoms is prerequisite for diagnosis) and the other group of 'dysthymia and adjustment disorders', which are characterised by the cognitive distortion. Until diagnostic practice is based on exact psychopathology, research will remain in its present state of confusion. For instance, the oft-repeated statement that cognitive therapy and biological treatments are of equal worth in the treatment of 'depression' will continue to be made. The statement may be true if no distinction is made between different depressive disorders but non-responders to the one or other treatment will have different characteristics: the psychotherapeutic approach will be more successful in the disorders based on cognitive distortion whereas the biological treatments are likely to be more effective when major depressive disorder is present.

Ayuso-Mateos, J. L., Vázquez-Barquero, J. L., Dowrick, C., et al (200I) Depressive disorders in Europe: prevalence figures from the ODIN study. British Journal of Psychiatry, 179, 308-316.

Snaith, P. (1993) What do depression rating scales measure? British Journal of Psychiatry, 163, 293-298.

Thompson, C., Ostler, K., Peveler, R. C., et al (200I) Dimensional perspective on the recognition of depressive symptoms in primary care. The Hampshire Depression Project 3. British Journal of Psychiatry, 179 317-323.

P. Snaith 30 Gledhow Wood Road, Leeds LS8 4BZ,UK. E-mail: psyrps@stjames. leeds.ac.uk

\section{Factor structure of the Hospital Anxiety and Depression (HAD) scale}

We would like to draw attention to the assertion by Mykletun et al (2001) that a two-factor structure best fits the Hospital Anxiety and Depression (HAD) scale, especially in individuals with mental problems. They stated that psychometric studies of this scale only involved small samples of non-psychiatric patients. However, we recently published the only factor analysis of the HAD scale based on a large population: 2669 'HAD completers' from 3002 patients $(89 \%)$ with major depression, DSM-IV criteria (Friedman et al, 2001).

Contrary to Mykletun et al, we found a three-factor solution using principalcomponents analysis with factors defined by eigenvalues $\geqslant 1$. One of Mykletun 
et al's reasons for rejecting the three-factor solution was that their third factor comprised heterogeneous items loading for both anxiety (items 7 and 11) and depression (item 14). Our three-factor structure discriminates the original depression factor and two separate constructs of anxiety: 'psychic anxiety' (items 3, 5, 9 and 13) and 'psychomotor agitation' (items 1, 7 and 11). This factor solution captured $48.6 \%$ of the variance and was relatively robust; it was not influenced by gender ratio and was also found in two random halves.

Two reasons may account for these discrepancies between our results. First, because of the high proportion of HAD scale non-completers (44\%), Mykletun et al's sample may have been biased. Patients with depression are probably not prone to answer such surveys and may therefore be underrepresented. Second, the factor structure of the HAD scale may not be stable across different categories of subjects: those with heterogeneous mental problems and those specifically suffering from major depression.

The HAD scale is not only useful for its initial screening purpose. It also showed potential ability in assessing change in specific symptoms of anxiety ('psychic anxiety' and 'psychomotor agitation' factors of the scale) during antidepressant treatment (Friedman et al, 2001). Moreover, recognition and monitoring of psychomotor agitation has several clinical implications: it is a potential side-effect of some antidepressants (Nutt, 1999), it may predict antidepressant response (Flament et al, 1999), it may predict adverse outcome and increase the risk of suicide (Schatzberg \& DeBattista, 1999).

\section{Declaration of interest}

S.F. has formerly been CNS medical adviser for Pfizer France; J-C.S. has received fees from Pfizer France; J.D.G. has received fees from several pharmaceutical companies.

\section{Flament, M. F., Lane, R. M., Zhu, R., et al (1999) Predictors of an acute antidepressant response to fluoxetine and sertraline. International Clinical Psychopharmacology, 14, 259-275.}

Friedman, S., Samuelian, J. C., Lancrenon, S., et a (200I) Three-dimensional structure of the Hospital Anxiety and Depression Scale in a large French primary care population suffering from major depression. Psychiatry Research, 104, 247-257.

Mykletun, A., Stordal, E. \& Dahl, A. A. (200I) Hospital Anxiety and Depression (HAD) scale: factor structure, item analyses and internal consistency in a large population. British Journal of Psychiatry, $\mathbf{1 7 9}$ 540-544.
Nutt, D. J. (1999) Care of depressed patients with anxiety symptoms. Journal of Clinical Psychiatry, $\mathbf{6 0}$ (suppl. 17), S23-S27.

\section{Schatzberg, A. F. \& DeBattista, C. (1999)}

Phenomenology and treatment of agitation. Journal of Clinical Psychiatry, 60 (suppl. 15), SI7-S20.

S. Friedman, C. Even Clinique des Maladies Mentales et de l'Encéphale (CMME), Centre Hospitalier Sainte-Anne, I rue Cabanis, 75674 Paris Cedex 14, France

J.-C. Samuelian CMU de la Timane, Aix Marseille II, France

J. D. Guelfi CMME, Centre Hospitalier SainteAnne, Paris, France

Authors' reply: Friedman et al raise doubts as to the two-factor structure of the HAD scale reported by us. The size of our sample $(n=51930)$ allowed us to test our finding in several sub-samples. Using principalcomponents analysis, the same two-factor solution was also found in all sub-samples reporting somatic and psychiatric problems, as well as in all age- and gendergroups from 20 to 89 years. This indicates that the two-factor structure of the HAD scale is robust and stable. Therefore, eventual minor biases due to response rates cannot account for the discrepancy between Friedman et al's and our findings. Our third factor, which emerged only in sub-samples with low depression scores, always showed a low eigenvalue. Our results are in accordance with the conclusions of a recent literature review on the HAD scale (Bjelland et al, 2002) which concludes that a two-factor solution is most commonly found.

Friedman et al (2001) have a sample $(n=2669)$ characterised by major depression (DSM-IV), which corresponds to high depression and probably variable anxiety scores on the HAD scale. When performing factor analysis, composition of the sample is essential for the results. If an inclusion criterion restricts the variance and covariance of the variables entered in the factor analysis, this will influence the factor solution found. The results by Friedman et al can be interpreted as a consequence of their restriction of their sample to major depression only, as this restricts the covariance between items on the HAD scale. In our sub-sample with various mental problems $(n=2098)$ the two-factor solution is robust with high explained variance $(82.1 \%)$.

Friedman $e t$ al's findings are of interest, however, since they answer the question:
What is the factor structure of the HAD scale when anxiety appears in major depression? Comparing the fit coefficients between two- and three-factor solutions using confirmatory factor analysis must show the advantage of a three-factor solution. Friedman et al seem to presume that the factor structure of anxiety found in major depression is identical to that found for anxiety in the general population.

The advantage of population samples is that selection bias is minimised. In several of our studies based on the unselected HUNTII population (from the Nord-Trøndelag Health Study) we have found results at variance with those of clinical samples (Engum et al, 2002; Wenzel et al, 2002). This could also explain the discrepancy between Friedman et al's and our results.

Bjelland, I., Dahl, A. A., Haug, T. T., et al (2002) The validity of the Hospital Anxiety and Depression Scale. An updated literature review. Journal of Psychosomatic Research, 52, 69-77.

Engum, A., Bjøro, T., Dahl, A. A., et al (2002) An association between depression, anxiety and thyroid function - a clinical fact or an artefact? Acta Psychiatrica Scandinavica, in press.

Friedman, S., Samuelian, J.-C., Lancrenon, S., et a (200I) Three-dimensional structure of the Hospital Anxiety and Depression Scale in a large French primary care population suffering from major depression. Psychiatry Research, 104, 247-257.

Wenzel, H. G., Haug, T. T., Mykletun, A., et al (2002) A population study of anxiety and depression among persons who report whiplash traumas. Journal of Psychosomatic Research, in press.

\section{A. A. Dahl, A. Mykletun, E. Stordal}

Department of Psychiatry, Aker University Hospital, N-0320 Oslo, Norway

\section{Follow-up of childhood depression: historical factors}

The study by Fombonne et al (2001), following adolescents with diagnoses of major depressive disorder into adulthood, raises some questions pertaining to the era when they were diagnosed (1970-1983).

First, it was only in the early 1980s that child abuse began to come into the awareness of professionals and, a few years later, the general public. Therefore, it is possible that some of the young people identified with depressive disorders may have had a history of sexual abuse which was not disclosed or enquired about. This raises the question of what would have been the outcome in those young people who had been sexually abused had they made disclosures and had appropriate therapeutic intervention for this. It is well known that 